\title{
Data Acquisition Process for an Intelligent Decision Support in Gynecology and Obstetrics Emergency Triage
}

\author{
*Alexandra Cabral , ,Carla Pina ${ }^{2}$, Humberto Machado ${ }^{3}$, António Abelha ${ }^{4}$, \\ *Maria Salazar ${ }^{1}$, *César Quintas ${ }^{1}$, Filipe Portela ${ }^{5}$, José Machado ${ }^{4}$, \\ José Neves ${ }^{4}$, Manuel Filipe Santos ${ }^{5}$ \\ ${ }^{1}$ Sistemas de Informação, ${ }^{3}$ Serviço Urgência, CHP - Hospital Santo António \\ Largo Prof. Abel Salazar, Porto, Portugal \\ ${ }^{1}$ alexandra, msalazar, cesar.quintas\}@chporto.min-saude.pt \\ 3idirector.su@chporto.min-saude.pt \\ ${ }^{2}$ Hospital São Sebastião, Santa Maria da Feira, Portugal, \\ carlampina@gmail.com \\ ${ }^{4}$ Dep. Informática, ${ }^{5}$ Centro Algoritmi - Dep. Sistemas de Informação, \\ Universidade do Minho, Portugal, \\ ${ }^{4}$ \{abelha, jmac, jneves\}@di.uminho.pt \\ ${ }^{5}\{\mathrm{cfp}, \mathrm{mfs}\} @$ dsi.uminho.pt
}

\begin{abstract}
Manchester Triage System is a reliable system of triage in the emergency department of a hospital. This system when applied to a specific patients' condition such the pregnancy has several limitations. To overcome those limitations an alternative triage IDSS was developed in the MJD. In this approach the knowledge was obtained directly from the doctors' empirical and scientific experience to make the first version of decision models. Due to the particular gynecological and/or obstetrics requests other characteristics had been developed, namely a system that can increase patient safety for women in need of immediate care and help low-risk women avoid high-risk care, maximizing the use of resources. This paper presents the arrival flowchart, the associated decisions and the knowledge acquisition cycle. Results showed that this new approach enhances the efficiency and the safety through the appropriate use of resources and by assisting the right patient in the right place.
\end{abstract}

Keywords: Triage; Emergency Department; Intelligent Decision Support Systems; Manchester Triage System

\section{Introduction}

Typically, in medical emergencies, triage is the process of determining the priority of patients treatments based on the severity of their clinical condition.

There are various types of triage systems and the most commonly used are those with five levels of severity, such as the Emergency Severity Index (ESI) [1], the Manchester Triage System (MST) [2] and the Canadian Triage and Acuity Scale (CTAS) [3]. 
Maternidade Júlio Dinis (MJD) is a maternity hospital that provides care for women during pregnancy and childbirth and for newborn infants. In the Emergency Department (ED) of MJD, women who seek Gynecological and/or Obstetrics (GO) emergency care (pregnant, non-pregnant, parous or primiparous) pass by a triage system specially developed for GO, which is presented in this paper.

Previously, they were also assisted in the ED of Hospital Geral de Santo António (HGSA) using the MTS, which hasn't been effective for GO due to the generality of the questions used for the triage. However, there was still the need for a triage system in MJD because the misclassification of non-urgent patients has been pointed as a problematic aspect in emergency settings when patients severity is not identified at triage, or if there is no accordance on what problems are non-urgent. [4-7].

Furthermore, there are numerous limitations associated with the process of validating triage scales. Even in developed countries, there are problems in conceptualizing validation methods [8]. Moreover, it is known that several maternal symptoms require special evaluation. It should also be stressed that the limited budgets for health care make crucial to prioritize patients' needs and assist them with the most appropriate resources.

The above pointed restrictions motivated the development of a triage system specific for GO that supports the decision-making process for a better healthcare in MJD by distinguishing urgent and non-urgent patients. An Intelligent Decision Support System (IDSS) is in construction to support this process through the use of the knowledge discovery and data mining techniques to predict the level of urgency and help to choose the better decision for each situation.

The paper is divided in five chapters. The first one introduces the MJD, the MST and the IDSS. The second one presents the actual context and the foundations for triage and how Manchester System works. The third chapter outlines the IDSS implemented for the GO purposes, the main objectives and changes occurred. An illustrative example is used to explain the GO Triage process. The fourth part of this paper presents some qualitative and quantitative results obtained so far. Finally, some discussions and conclusions are made.

\section{Background and related work}

\subsection{Context}

MJD is part of Centro Hospitalar do Porto (CHP), together with Hospital de Santo António (HSA) and Hospital Maria Pia (HMP). CHP was created in 2007 and, prior to that, the three were separated entities. Women in need of urgent Care (GO) could attend the ED of either HGSA or MJD. Since the creation of CHP, women in such conditions are encouraged to attend the ED of MJD instead of HSA (ex-HGSA).

HSA has a general ED working with the MTS since 2000 and, to optimize the care provided to women in urgent or emergent situations, they are sent to the ED of MJD with a specific triage system that we developed, which is in use for six months, since January of 2010. 


\subsection{Intelligent Decision Support System}

According to Turban [9], a decision support system is an interactive, flexible and adaptable information system, developed to support a problem solution and to improve the decision making. These systems usually use artificial intelligence techniques and are based on prediction and decision models that analyze a vast amount of variables to answer a question.

The decision making process can be divided in five phases: intelligence, design, choice, implementation and monitoring [9]. To be an IDSS there exist some features that have to be accomplished. [10] The presence of an IDSS on an ED permits a better understanding of the real state of the patient and can improve the output results, because it can help the nurses giving the best care to the women on the time that they needs.

\subsection{Triage}

Triage is a process developed with the agreement of a panel of experts based on decision rules and is an integral part of the modern ED [11]. It is mostly supported by computerized information systems [12].

In the triage process, decision-making involves the interpretation, discrimination and evaluation of communication between the patient and the health care providers that are carrying out the triage process [13].

Good decision making is crucial because patient outcome is greatly influenced by the initial assessment of the triage. The main method used in the triage process by the professional community in HSA is the MTS, which is described in the next section.

Additionally, it is explained why this system does not fit urgent and emergent situations for $\mathrm{GO}$ and is presented the system we developed for that purpose.

Obstetric triage has been one of the latest obstetric services to emerge throughout the last decade and hospitals have incorporated triage principles into the practice of $\mathrm{GO}$ [14].

GO triage occurs in a women's hospital emergency unit, where a variety of GO conditions are presented, since labor assessment and assistance to common GO conditions. It is, therefore, of great importance to ensure that women who attend the triage unit are properly evaluated and that high-risk patients are accordingly treated, not just the mother, but also the fetus.

According to the severity level allotted to the patients in the ED, they are assigned to the appropriate observation area and the first observation should occur in a defined period of time.

The purposes of ED triage are described as "assigning the patient a place in queue, assigning an area or treatment room, predicting resource consumption and identifying non urgent patients who could be diverted to other, presumably less expensive venues" $[15,16]$. Likewise, triage for GO has the ability to screen, prioritize, and expedite GO conditions, and is used to rate the severity of women's condition and to optimize the resources available, for the resources of ED can be overused for nonurgent patients. This ability also lends itself to the emergent care skills needed in an obstetric triage unit as well as effective triaging [17]. 
In conclusion, the main concerns in triage, either in GO or in other medical specialties are to improve the quality of care and reduce the risks arising from the waiting time to emergent care. Discrepancies in triage may lead to inappropriate use of resource and may contribute to both patient and staff dissatisfaction.

"If overtriage occurs, excessive resources are used for patients with nonurgent problems, resulting in excess costs and delays of care for the patients with more severe problems. If undertriage occurs, potentially sick patients may be triaged as nonurgent resulting in a clinically unsafe diversion from the ED." [4].

\subsection{Manchester Triage System}

The Manchester Triage System (MTS) [2] is a five point triage scale used to triage patients presenting to the ED, which was introduced in the United Kingdom in 1996 and is now widespread, especially in Europe, and has been in use in HSA since 2000.

Although the MTS is a reliable system of triage in the ED, well suited for general emergency situations, in particular cases like the GO care, its use is unsatisfactory due to the generalization of the questions it uses for classifying patients' clinical severity status. This system workflow confines to a number of presenting problems with associated flowcharts and supporting documentation. It has five levels of classification. Table 1 presents the five levels of classification of the MTS and the corresponded recommended waiting times for patients.

Table 1. - MTS levels and recommended waiting times [18]

\begin{tabular}{cc}
\hline Levels & Patient should be seen by provider within \\
\hline 1 - Immediate & 0 minutes \\
2 - Very urgent & 10 minutes \\
3 - Urgent & 60 minutes \\
4 - Standard & 120 minutes \\
5 - Nonurgent & 240 minutes \\
\hline
\end{tabular}

The benefits of the MTS were examined and considered to overcome other systems [13]. Some advantages were identified, highlighting the fact that the MTS is a method of triage internationally recognized, reliable and professionally evaluated and successfully used in a number of different health care systems.

Although the literature states that the MTS is a suitable evidence-based triage method and could be expanded to include other EDs of band one hospitals [13], it is not suitable to ED for GO, as experienced in HSA. Discussions arose that led to the conclusion that the criteria document that supports the MTS system did not satisfy the requirements for emergency care in GO. 
MTS is simple enough to allow a quickly assess of the patients' clinical condition and is applicable for the wide-range population with a large variability in signs and symptoms in the emergency care setting [11].

Therefore, its use is more suitable for general medical emergencies and not the specific emergencies of women in need of GO medical assistance.

Furthermore, the discriminatory power of MTS is not equal for medical and surgical specialties, which may be related to the character of its natural discriminators [19].

Triage is a process developed with the agreement of a panel of experts based on decision rules and is an integral part of the modern ED [11]. It is mostly supported by computerized information systems [12].

In the triage process, decision-making involves the interpretation, discrimination and evaluation of communication between the patient and the health care providers that.

\section{Triage system for gynecology and obstetrics in MJD}

Prior to the creation of CHP, pregnant women and women in need of urgent or emergent care were admitted in HGSA and MJD for emergency situations. In HGSA, the MTS had been used since 2000. However, the healthcare professionals realized that the MTS was not adapted to the GO service due to the degree of generalization of the questions. Moreover, more than $40 \%$ of the cases were not emergencies, which would translate in a misuse of resources. Accordingly, we developed a triage system specific for $\mathrm{GO}$, where the questions are focused on that particular type of patients, i.e. pregnant or parous women. After the creation of CHP, GO emergencies were transferred to MJD with the new triage system.

As depicted in the graph of the Fig. 1, a decrease of $85 \%$ in GO emergencies episodes occurred in the first six months of 2010 when compared with the same period of 2009.

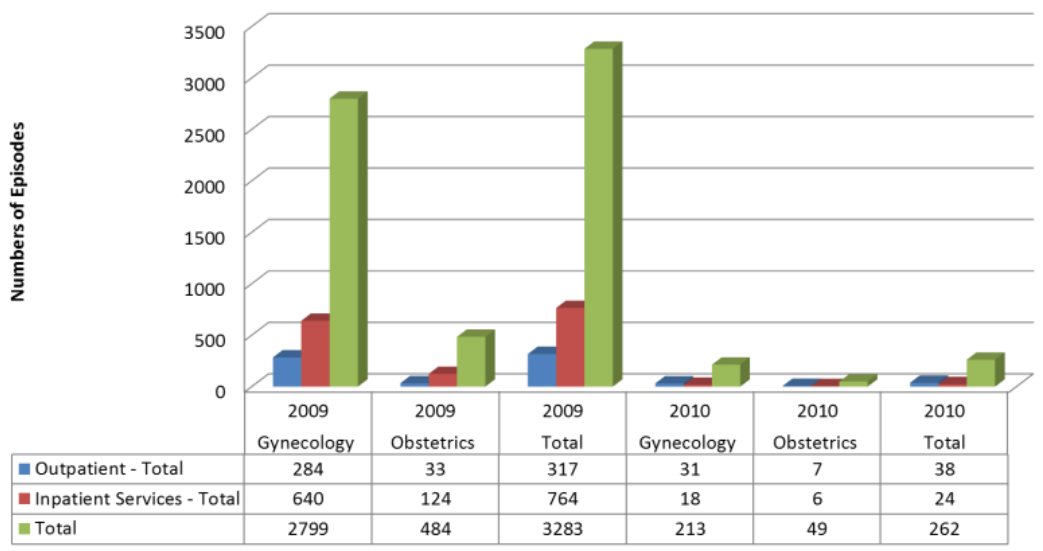

Fig. 1. GO Episodes in CHP 
We developed an effective triage model that meets the needs of the ED for GO, particularly a system that can increase patient safety for women in need of immediate care (proving) and help low-risk women avoid high-risk care, maximizing the use of resources.

The goal of this system is to classify patients according to the severity of their clinical condition, establishing clinical priorities and not diagnosis.

The triage is done by specialized physicians and is based on a set of predefined questions in the form of rules of a decision tree. According to the result, the IDSS indicates whether the patient should be sent to an urgent (URG) or normal consultation (ARGO - Outpatient Clinic). Women in need of urgent care (e.g. women who arrive by ambulance in very serious conditions) are immediately assisted, without any triage.

Before, in MJD, patients were admitted in the order of their arrival, not differentiating the cases that required immediate or intermediate assistance and the non-urgent cases. In HGSA they were classified with the MTS.

The IDSS brought some improvement in healthcare, essentially because:

- Contributes to the decrease of clinical errors;

- Provides cost-effective and proper care;

- Improves client satisfaction.

Moreover, by transferring all the GO emergencies to MJD and correctly assessing their severity, it also contributes for reducing overcrowding of ED [20-22]

When women arrive at the MJD requiring urgent observation, and before any admission paperwork, a physician makes the triage using the system. It indicates if the patient should be assisted with urgency or be sent to a normal first medical appointment.

In opposition to the MTS, triage is done by physicians and not by nurses. In general ED triage, nurses usually gather the clinical information and make the triage decision.

However, tests in MJD showed a lack of agreement in triage assessment between nurses and doctors. Moreover, a study showed that the triage decisions physicians make are significantly different when they have and take the opportunity to visually evaluate patients [23].

Accordingly, a visual assessment can enhance the doctors' intuitive ability to quickly distinguish a sick from a not-so-sick patient. In conclusion, close supervision by a doctor is essential because of the significant risks involved [24].

The flowchart (fig. 2) describes the real-time decision process, in ED, after some woman arrive to MJD. 


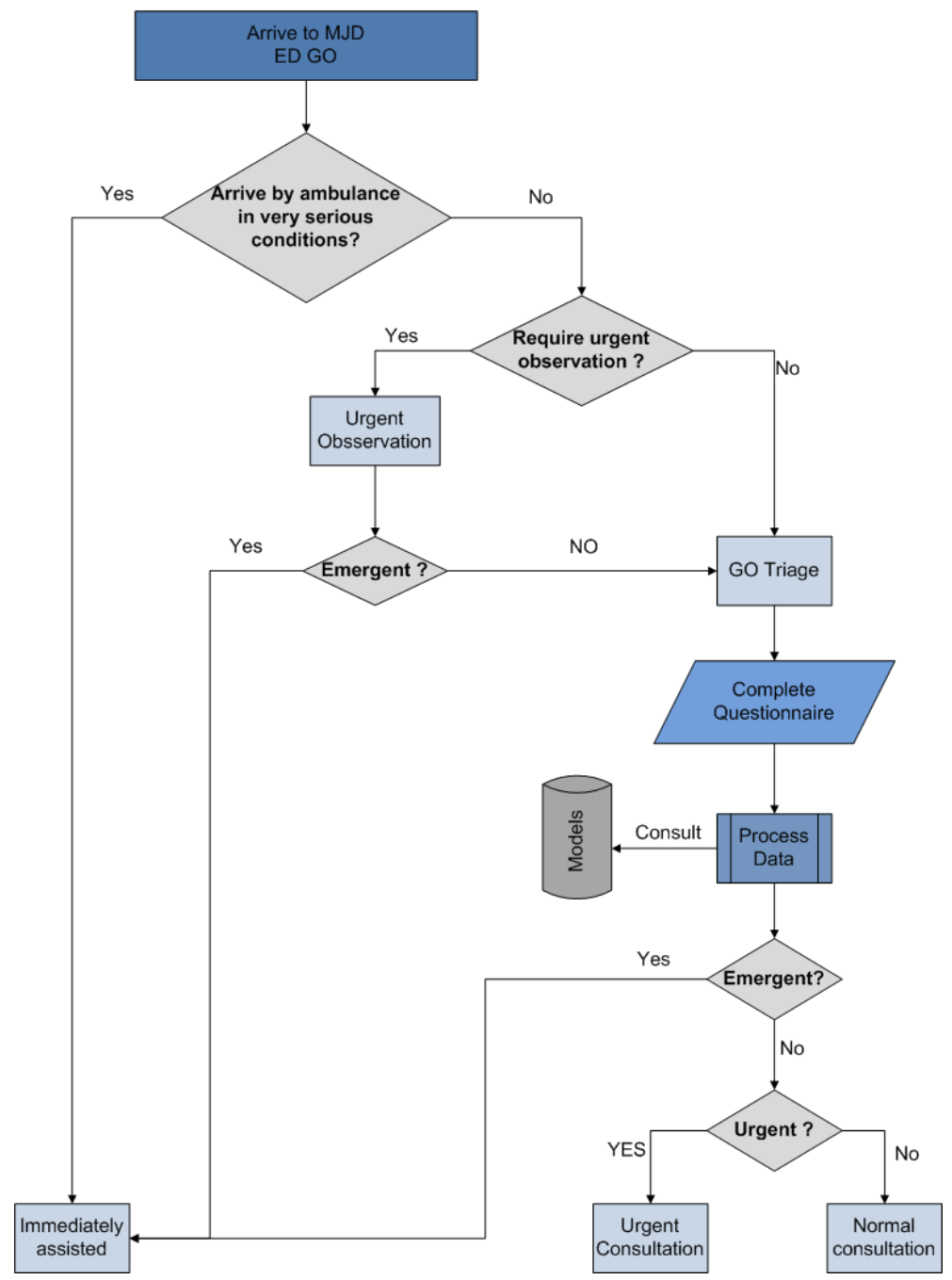

Fig. 2. GO process

In the future we will use the results of questionnaires and consultation and the decisions made during the experimental period to create prediction models in order to accurately discriminate the urgent cases, dividing the actual urgent class in two subclasses: urgent and can be urgent.

The last type of patients (can be urgent) will be directed to a normal consultation. During the waiting time they will be in observation and if something happens aggravating the clinical condition, the priority will be changed to urgent and the patient will be immediately assisted. 


\section{Results}

The system has been at work since January of 2010. In six months, 9959 women passed by the triage system. Figure 3 shows the distribution of the population by the two possible categories of the triage - ARGO and URG.

Accordingly, almost $50 \%$ of the cases (4299) were classified as non-urgent (ARGO) (FIG.3), which means that those women were sent to a normal first medical appointment, reducing ED overcrowding and contributing to a better efficiency.

Without this system, all women would have been assisted with urgency, according to their time of arrival, not the severity (or lack of) of their clinical condition.

The effects of this scenario would be misuse of resources and potentially more urgent cases could be assisted after the less urgent. It is also worth noting that, in six months, near 10000 women was assisted in MJD, contributing to less crowding in the ED of HSA.

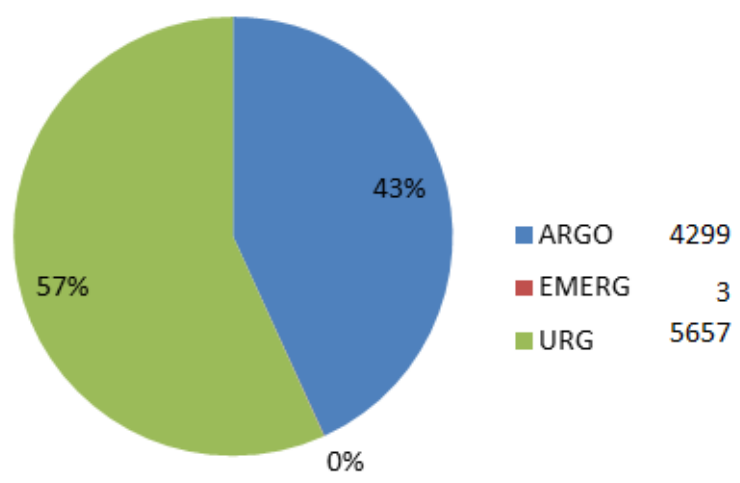

Fig. 3. Results of the triage system

Fig. 4 shows the distribution of the possible causes pointed by the patients as the motivation to come to MJD. As we can see 35\% of these women are not pregnant and some of those women need to be observed in other units like Institute of Gynecology and Obstetrics (IGO).
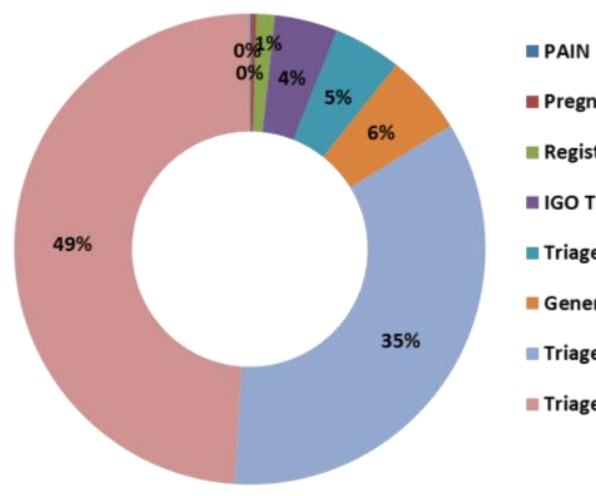

- Pregnant, Reason for Visit

= Register Triage

- IGO Triage

- Triage of Pregnant Women for CTG

m General

= Triage of not Pregnant Women

= Triage of Pregnant Women

Fig. 4. MJD Triage type 
The modification introduced in the mode the triage was done increased the number of the first consults reducing the waiting list.

This improvement turned CHP eligible to receive a money prize, by the Health Ministry, as a performance bonus to ensuring greater accessibility to special care (Outpatient Clinic).

\section{Discussion}

Even though it is a two-level system, in opposition to the prior system with a fivescale (MTS), the new system has proven to be more effective and brought benefits in healthcare of GO emergencies, in comparison to the previous one, where the attendance was done by arrived order and however sometimes was analyzed the clinical risk.

Patient priority is defined by the classes' urgent or first medical appointment. Although it is not a class, emergent cases have the highest priority and are sent immediately for the observation room, with no need for triage.

With this strategy it is guaranteed that resources are optimally used and that women are assisted accordingly to the urgent degree of their medical condition, which has been pointed as major issues in triage systems.

\section{Conclusion}

Some important changes were made. With this system the ED professionals can better assist the patients according to their urgency i.e. if some case is really urgent (emergent) is because is very danger to patient health and will be treated instantly.

With these options we can have three types of patients: Emergent, Urgent or Normal, and they will be observed / attended with the level of risk. The IDSS help the nurses to make the better decision according some decision rules that in the future will be integrated in the prediction models.

With this changes we can prove that the use of clinical knowledge of this specific area allow to have a system more suited to this type of users, contrary to what it happened previously.

Like we showed GO benefits from the adoption of the IDSS. The future work includes the use of the data collected to optimize the rules and the data mining models as a way to improve the IDSS performance and to generate more accurate knowledge.

\section{References}

1. Tanabe, P., Gimbel, R., Yarnold, P.R., Adams, J.G.: The Emergency Severity Index (version 3) 5-level triage system scores predict ED resource consumption. J Emerg Nurs 30, 22-29 (2004)

2. Mackway-Jones, K., Marsden, J., Windle, J.: Emergency triage: Manchester triage group. BMJ (1997) 
3. Beveridge, R., Ducharme, J., Janes, L., Beaulieu, S., Walter, S.: Reliability of the Canadian Emergency Department Triage and Acuity Scale: Interrater Agreement. Annals of Emergency Medicine 34, 155-159 (1999)

4. Wuerz, R., Fernandes, C., Alarcon, J.: Inconsistency of emergency department triage. Annals of emergency medicine 32, 431-435 (1998)

5. Hayden, S.R., Jouriles, N.J., Rosen, P.: Requiem for "Non-Urgent" Patients in the Emergency Department. The Journal of Emergency Medicine 38, 381-383 (2010)

6. Rassin, M., Nasie, A., Bechor, Y., Weiss, G., Silner, D.: The characteristics of self-referrals to ER for non-urgent conditions and comparison of urgency evaluation between patients and nurses. Accident and Emergency Nursing 14, 20-26 (2006)

7. Bianco, A., Pileggi, C., Angelillo, I.F.: Non-urgent visits to a hospital emergency department in Italy. Public Health 117, 250-255 (2003)

8. Twomey, M., Wallis, L.A., Myers, J.E.: Limitations in validating emergency department triage scales. Emergency Medicine Journal 24, 477 (2007)

9. Turban, E., Aronson, J.E., Liang, T.-P.: Decision Support Systems and Intelligent Systems. Prentice Hall (2005)

10. Portela, F., Santos, M., Vilas-Boas, M., Rua, F., Silva, Á., Neves, J.: Real-time Intelligent decision support in intensive medicine. KMIS 2010- International Conference on Knowledge Management and Information Sharing, pp. 7, Valência, Espanha (2010)

11. Moll, H.A.: Challenges in the validation of triage systems at emergency departments. Journal of clinical epidemiology (2009)

12. Dong, S.L., Bullard, M.J., Meurer, D.P., Blitz, S., Ohinmaa, A., Holroyd, B.R., Rowe, B.H.: Reliability of computerized emergency triage. Academic Emergency Medicine 13, 269-275 (2006)

13. Cronin, J.G.: The introduction of the Manchester triage scale to an emergency department in the Republic of Ireland. Accident and Emergency Nursing 11, 121-125 (2003)

14. PSRS: The Pennsylvania Patient Safety Advisory. vol. V, pp. 110, Pennsylvania (2008)

15. Derlet, R.W., Kinser, D., Ray, L., Hamilton, B., McKenzie, J.: Prospective identification and triage of nonemergency patients out of an emergency department: a 5-year study. Annals of emergency medicine 25, 215-223 (1995)

16. Trzeciak, S., Rivers, E.P.: Emergency department overcrowding in the United States: an emerging threat to patient safety and public health. British Medical Journal 20, 402 (2003)

17. JáAngelini, D.: Obstetric triage: the realm of the midwife part 2. Journal of NurseMidwifery 44, 536 (1999)

18. http://www.ahrq.gov/research/esi/esitab2-2.htm

19. Martins, H.M.G., De Castro Dominguez Cuña, L.M., Freitas, P.: Is Manchester (MTS) more than a triage system? A study of its association with mortality and admission to a large Portuguese hospital. Emergency Medicine Journal 26, 183 (2009)

20. Olshaker, J.S.: Managing emergency department overcrowding. Emerg Med Clin North Am 27, 593-603 (2009)

21. Solberg, L.I., Asplin, B.R., Weinick, R.M., Magid, D.J.: Emergency department crowding:: Consensus development of potential measures. Annals of emergency medicine 42, 824-834 (2003)

22. Johnson, M., Myers, S., Wineholt, J., Pollack, M., Kusmiesz, A.L.: Patients who leave the emergency department without being seen. Journal of Emergency Nursing 35, 105-108 (2009)

23. Brillman, J.C., Doezema, D., Tandberg, D., Sklar, D.P., Skipper, B.J.: Does a physician visual assessment change triage?* 1 . The American journal of emergency medicine 15,29 33 (1997)

24. Bygaryventolini, M.D., Ranneiger, M.D.: Avoiding the pitfalls of obstetric triage. OBG MANAGEMENT (2003) 